\title{
Editorial: Supramolecular Nanomaterials for Engineering, Drug Delivery, and Medical Applications
}

\author{
Elise Lepeltier ${ }^{1}$, Vincent Levet ${ }^{2}$, Tu Lee ${ }^{3}$, Nathalie Mignet ${ }^{4}$, Jianliang Shen ${ }^{5}$, \\ Hicham Fenniri ${ }^{6 *}$ and Yohann Corvis ${ }^{4 *}$ \\ ${ }^{1}$ Micro et Nanomédecines Translationnelles, MINT, UNIV Angers, Inserm 1066, CNRS, Angers, France, ${ }^{2}$ GSK Vaccines, Rue \\ de l'Institut 89, Rixensart, Belgium, ${ }^{3}$ Department of Chemical and Materials Engineering, National Central University, Taoyuan \\ City, Taiwan, ${ }^{4}$ Université de Paris, CNRS, Inserm, UTCBS, Chemical and Biological Technologies for Health Group \\ (utcbs.cnrs.fr), Faculté de Pharmacie, Paris, France, ${ }^{5}$ School of Ophthalmology \& Optometry, School of Biomedical \\ Engineering, Wenzhou Medical University, Wenzhou, China, ${ }^{6}$ Departments of Chemical Engineering, Bioengineering, \\ Chemistry \& Chemical Biology, Northeastern University, Boston, MA, United States
}

Keywords: bioinspired nanomaterials, drug delivery and targeting, vaccines production and development, diagnostic agents, cancer treatment

\section{Editorial on the Research Topic}

Supramolecular Nanomaterials for Engineering, Drug Delivery, and Medical Applications

\section{OPEN ACCESS}

Edited and reviewed by: Tony D. James,

University of Bath, United Kingdom

${ }^{*}$ Correspondence: Hicham Fennir

h.fenniri@northeastern.edu Yohann Corvis

yohann.corvis@u-paris.fr

Specialty section:

This article was submitted to

Supramolecular Chemistry,

a section of the journal

Frontiers in Chemistry

Received: 05 November 2020 Accepted: 11 November 2020 Published: 09 December 2020

Citation:

Lepeltier E, Levet V, Lee T, Mignet N, Shen J, Fenniri H and Corvis Y (2020) Editorial: Supramolecular Nanomaterials for Engineering, Drug Delivery, and Medical Applications. Front. Chem. 8:626468. doi: 10.3389/fchem.2020.626468
Programmed self-assembly and self-organization of carefully designed molecular monomers (Imai et al., 2018) has been widely explored to engineer stable nanostructures with the desired architecture and unique functionality (Lehn, 2015, 2017). This bottom-up approach could not only overcome design barriers associated with traditional molecular manufacturing at the nanoscale, but it could also endow the desired assemblies with adaptability, tunability, and stimuli-responsiveness due to the dynamic nature of the non-covalent interactions holding the architecture together. Hence, these supramolecular architectures may constitute the basis for novel smart nanomaterials with improved properties such as in vitro and in vivo physicochemical stability (Park et al., 2007), efficiency via drug loading improvement (Ahmed et al., 2019), exogenous environment adaptability (Pedersen et al., 2020), higher safety (Martin et al., 2020), manufacturability (Wren et al., 2020), and may have a broad range of applications with various interfaces, i.e., liquid/liquid (Prevot et al., 2018), solid/liquid (Couillaud et al., 2019), and gas/liquid (Manta et al., 2016; Corvis et al., 2018). Indeed, self-assembled systems (Beingessner et al., 2016; Mohamed et al., 2019) have been developed and widely explored in drug delivery (Chen et al., 2011; Song et al., 2011; Desmaële et al., 2012; Mignet et al., 2012; Al Sabbagh et al., 2020), gene delivery (Manta et al., 2017; Do et al., 2019), biomedical engineering (Sun et al., 2012; Childs et al., 2013; Meng et al., 2013; Puzan et al., 2018; Zhou et al., 2020), medicine (Journeay et al., 2008, 2009; Sun et al., 2014), and diagnostics. This body of work has led to the emergence of the field of supramolecular nanomedicine, which is the focus of this Research Topic for Frontiers in Chemistry (Figure 1).

The present Research Topic highlights nanomaterials importance in biological sciences through the supramolecular chemistry prism. Zhang et al. engineered dynamic hydrogels by cross-linking of O-carboxymethyl chitosan with reversibly connected imino-PEGylated dynamers. The double imine chitosan/dynamer and dynamer bonds were considered to provide tangled structures for controlled drug release behavior from the hydrogels. The structural and physical properties of the resulting hydrogels were examined, showing good thermal stability and optimized swelling behavior. When hydrogels with various composition ratios were further developed for the delivery of the anti-cancer drug fluorouracil (5-FU), high drug encapsulation rates up to $97 \%$ were obtained. 


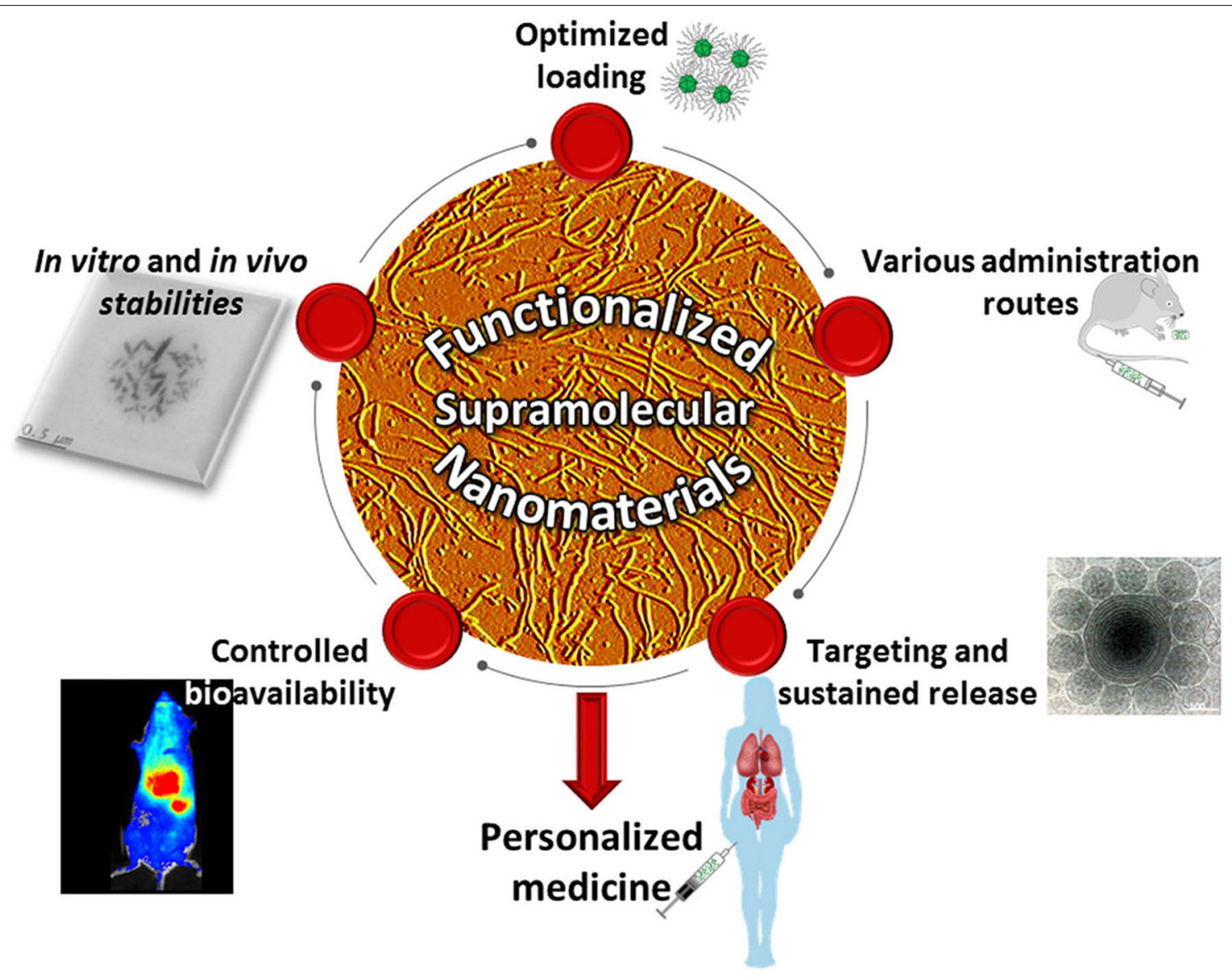

FIGURE 1 | Supramolecular nanomaterials and their applications. Highlight of the fields covered by this Research Topic.

5-FU in-vitro drug-release profiles from the hydrogels showed an initial burst-effect, followed by a slower increase and plateau. All reached a high total release within $12 \mathrm{~h}$, which varied upon the cross-linking level that depended on drug/carrier ratios and gelation temperature, thus demonstrating the potential adjustable sustained release applications of this new type of carrier, notably for topical administration.

However, exogen compounds even at the nanoscale dimension, have the propensity to be recognized by the mononuclear phagocyte system once in the bloodstream, inducing rapid clearance of the antigen from the blood and limiting the sustained release advantage. In order to prolong systemic circulation and to prevent the therapeutic agent from aggregation, opsonization or phagocytosis, nanoparticles have been coated with hydrophilic biocompatible co-polymers since the 1990's (Klibanov et al., 1990; Tan et al., 1993; Fam et al., 2020). Liu et al. are reporting surface functionalized Persistent Luminescence NanoParticles (PLNPs) which have been proved promising nanomaterials for bioimaging applications. For that purpose, poly(N-2-hydroxypropyl)methacrylamide (pHPMA), a hydrophilic polymer widely used in nanomedicine has been intended as an alternative strategy to coat $\mathrm{ZnGa}_{2} \mathrm{O}_{4}$ :Cr NPs for in vivo imaging. The results demonstrated that the newly designed ZGO NPs can circulate in mouse bloodstream much longer than non-coated $\mathrm{ZGO}-\mathrm{OH}$, i.e., at least an hour, without being trapped in the liver or spleen. The new strategy presented allowed to improve nanoparticules already developed by the same group for stability in lysosomal-like medium (Lecuyer et al., 2020) and luminescence efficiency (Maldiney et al., 2014; Teston et al., 2018).

A given nanomaterial obtained by supramolecular interactions may present various potential applications due to the intermolecular non-covalent forces. As outlined by Lu et al., supramolecular materials assembled may present interesting applications in various biomedical fields by integrating multiple interactions of polyphenols, especially tannic acid, a class of plant-derived biocompatible and biodegradable compounds consisting of two or more phenolic units in structure. Starting with the origin of supramolecular assembly, combined with the structural characteristics, biological activity of polyphenol and unique advantages of supramolecular assembly, this review systematically covers the classification of supramolecular assembly, assembly system and applications of tannic acid in, e.g., drug delivery, theranostics for cancer treatment, bone 
tissue engineering, and biofunctional membrane material. Finally, current and future challenges remaining in this field were put forward. In addition, some traditional or novel assembly techniques were introduced, which also provides some inspiration for the research in this field. Moreover, from a fundamental point of view, the investigation and applications of such dynamic systems could advance our understanding of the design and function of complex chemical and biological systems.

Complex systems can be obtained when ingredients are not in their thermodynamically stable state, e.g., for pharmaceutical liquid forms when drug overall quantity impose by the loading capacity (LC) is higher than its solubility in the same continuous medium, and/or when ingredients that are normally nonmiscible are associated together in the same formulation. In such "unstable" systems, excipients are added to kinetically stabilize these ingredients post self-assembly. Ho et al. described the synthesis and characterization of 4 different amphiphilic squalenyl derivatives (i.e., 1 anionic squalenyl derivative, 1 cationic squalenyl-ethanolamine derivative and 2 PEG-squalenyl derivatives of 750 and 3,000 Da, respectively), as well as the physicochemical and biopharmaceutical characterizations of their drug-loaded self-assembled NPs $(113-325 \mathrm{~nm})$, in aqueous solution, as drug delivery systems. Seven different model drugs, chosen to exhibit various physicochemical properties (i.e., hydrophobic, hydrophilic, charged) where used with different methods of nanoprecipitation. The best performing methods were determined for each type of formulated system (co-precipitation for hydrophobic compounds, solvent evaporation for hydrophilic compounds, dropping for small and high molecular weight hydrophilic compounds), in relation to the drug properties (molecular weight or $\log \mathrm{P}$ values). All NP derivatives demonstrated high LC up to $45 \% \mathrm{w} / \mathrm{w}$, excellent encapsulation efficiency up to 92\%, appropriate biocompatibility, adequate colloidal stability in a variety of physiological environments, and sustained release properties exhibiting very low burst-effect and slow controlled-release for at least $24 \mathrm{~h}$. These attributes were achieved thanks to modulated based loading method and loaded NP properties.

Sometime, natural amphiphilic stabilizing agents that are approved by FDA, like silk fibroin, may be preferred as nanocarriers for safety reasons. In their mini review, Ma et al. present the structures of silk fibroin, the controlled transformation of secondary structures, and the formation mechanism of silk fibroin-based nanoparticles and discuss the intrinsic multi-responsibility, surface functionalization, and transgenic modification of these systems for drug delivery. The silk fibroin-based NPs not only have the merits of excellent biocompatibility, but also show the features of multi-responsive systems thanks to stimuli such as $\mathrm{pH}$, hyperthermia, lysosomal enzyme, light, and reactive oxygen species. The multi-responsive phenomenon is helpful for ondemand drug release, reducing systemic side toxicities of some drugs.

Through the non-covalent interactions present in selfassembled therapeutic agents, one of the advantages of smart nanomaterials is that they can be adapted to a specific pathology for personalized medicine purpose. Feng et al. synthesized a novel diblock polymer consisting of polyethylene glycol and poly(glutamic acid (3-(2-nitro-imidazolyl)-propyl)) for hypoxia-responsive polymeric micelle self-assemblies allowing biocompatibility of blank micelles, a drug loading of $\sim 4 \%$, encapsulation efficiency of $\sim 42 \%$, and controlled release properties when loaded with the anticancer agent doxorubicin. The cell experiments ultimately demonstrated that drug-loaded micelles had a stronger apoptosis capacity on tumor cells under hypoxic conditions. All the experiments indicated that latter hypoxia-responsive polymeric micelles have a potential for enhanced cancer treatment since both the rapid and important growth and the relatively insufficient blood supply of the tumor tissues give rise to hypoxic conditions, compared with normal tissues.

However, nanomedicines are not only useful for small organic and inorganic molecules vectorization but also for gene delivery as investigated by Gaillard et al.. They developed dual targeting agents combining the mechanisms of bioactive alkylphospholipids and gene therapies. To achieve this goal, three prodrugs series were prepared based on three cytotoxic alkylphospholipids, namely miltefosine, perifosine, and erufosine. The prodrugs were selectively hydrolyzed at physiological $\mathrm{pH}$ to parent cytotoxic drugs. Lipoplexes of the prodrugs with plasmid DNA could transfect cancer cells and produced some enhancement of antiproliferative activity. Therefore, the authors prove that non-classical anti-cancer systems can be also designed by supramolecular formulation of nanomedicines.

To complete the insight into self-assembled nanosystems for treatment and diagnosis applications, supramolecular self-assembled peptide-based vaccines may be considered. Indeed, vaccination is considered, especially these days more than ever due to the coronavirus pandemic, as one of the greatest contributions of science to global health, remarkably through the eradication of great illnesses like smallpox and rinderpest and through its ability to control diseases such as measles and polio (Greenwood, 2014). Since the beginning, vaccination moved from antigens based on attenuated or inactivated viruses to purified proteins and more recently to peptide sub-unit epitopes. The latter are more and more recognized as one of the safest approach relative to control over manufacturing processes, contaminations, undesirable effects and autoimmune responses but sometimes comes at the cost of insufficient biological response. As an alternative, nanovaccines including supramolecular selfassembled peptide-based vaccines may be considered but their development is currently limited by our comprehension of selfassembled nanostructures and immune cells interactions (Negahdaripour et al., 2017; Fadeel, 2019). Advanced engineering of supramolecular assembly composed for instance of cell-penetrating peptides (Bechara and Sagan, 2013; Almeida et al., 2016) as delivery vehicles may help promoting a strong cellular or humoral immune response as they favorize targeted intracellular delivery of antigens. In the present Research Topic, Abudula et al. review the span 
of strategies applicable to constructing and applying such self-assembled structure as antigens themselves, going from their supramolecular architecture to their multiple application in vaccinology. The mechanisms behind the ability of these peptide-based nanovaccines to generate cell-mediated immunity or humoral response and the aspect of self-adjuvantation are highlighted. Finally, the challenges, clinical translatability and future perspectives are also discussed, considering the great potential of these innovative type of constructs for the future of vaccinology.

Self-assembled systems based on small molecules, block copolymers, dendrimers, peptides, and amphiphiles have been successfully synthesized to achieve a biological, medical, delivery, and/or sensing function(s). Some of these assemblies are designed to be responsive to stimuli such as ultrasound, light, $\mathrm{pH}$, temperature, or specific reagents. Because of their design features and current applications, these supramolecular nanosystems belong to the new and very active field of supramolecular nanomedicine. We thank the 59 co-authors all around the globe which have contributed to this Research Topic. Our main objective is to provide scientists, engineers and clinicians better and newer definition of nanomedicine through the publication of original papers or timely reviews covering synthetically accessible and chemically tunable self-assembled nanosystems endowed with a pharmaceutical, medical, biological, or diagnostic function.

\section{REFERENCES}

Ahmed, S., Corvis, Y., Gahoual, R., Euan, A., Lai-Kuen, R., Couillaud, B. M., et al. (2019). Conception of nanosized hybrid liposome/poloxamer particles to thicken the interior core of liposomes and delay hydrophilic drug delivery. Int. J. Pharm. 567:118488. doi: 10.1016/j.ijpharm.2019.118488

Al Sabbagh, C., Seguin, J., Agapova, E., Kramerich, D., Boudy, V., and Mignet, N. (2020). Thermosensitive hydrogels for local delivery of 5-fluorouracil as neoadjuvant or adjuvant therapy in colorectal cancer. Eur. J. Pharm. Biopharm. 157, 154-164. doi: 10.1016/j.ejpb.2020.10.011

Almeida, C., Lamaziere, A., Filleau, A., Corvis, Y., Espeau, P., and AyalaSanmartin, J. (2016). Membrane re-arrangements and rippled phase stabilization by the cell penetrating peptide penetratin. Biochim. Biophys. Acta Biomembr. 1858, 2584-2591. doi: 10.1016/j.bbamem.2016.07.012

Bechara, C., and Sagan, S. (2013). Cell-penetrating peptides: 20 years later, where do we stand? FEBS Lett. 587, 1693-1702. doi: 10.1016/j.febslet.2013.04.031

Beingessner, R. L., Fan, Y., and Fenniri, H. (2016). Molecular and supramolecular chemistry of rosette nanotubes. RSC Adv. 6, 75820-75838. doi: $10.1039 / \mathrm{c} 6 \mathrm{ra} 16315 \mathrm{~g}$

Chen, Y., Song, S., Yan, Z., Fenniri, H., and Webster, T. J. (2011). Self-assembled rosette nanotubes encapsulate and slowly release dexamethasone. Int. J. Nanomed. 6, 1035-1044. doi: 10.2147/ijn.s18755

Childs, A., Hemraz, U. D., Castro, N. J., Fenniri, H., and Zhang, L. G. (2013). Novel biologically-inspired rosette nanotube plla scaffolds for improving human mesenchymal stem cell chondrogenic differentiation. Biomed. Mater. 8:065003. doi: 10.1088/1748-6041/8/6/065003

Corvis, Y., Manta, S., Thebault, C., Couture, O., Dhotel, H., Michel, J.P., et al. (2018). Novel perfluorinated triblock amphiphilic copolymers for lipid-shelled microbubble stabilization. Langmuir 34, 9744-9753. doi: 10.1021/acs.langmuir.8b01668

Couillaud, B. M., Espeau, P., Mignet, N., and Corvis, Y. (2019). State of the art of pharmaceutical solid forms: from crystal property issues to nanocrystals formulation. ChemMedChem 14, 8-23. doi: 10.1002/cmdc.201800612

\section{DEDICATIONS}

This article is dedicated to Dr. Hai Doan Do, UTCBS' angel who left us too soon.

\section{AUTHOR CONTRIBUTIONS}

HF and YC have proposed the idea of this Research Topic. All authors contributed to the guest editorial board and the related manuscript has been read and revised by all the authors.

\section{FUNDING}

This work was financially supported by CNRS, Inserm, French Ministry of Higher Education Research and Innovation, National Natural Science Foundation of China (31800833 and 21977081), and Zhejiang Provincial Natural Science of Foundation of China (LZ19H180001).

\section{ACKNOWLEDGMENTS}

NM and YC would like to thank Dr. Cyrille Richard, Dr. Johanne Seguin, Dr. Thomas Lecuyer, Dr. Brice Martin, Dr. Shayan Ahmed, Jianhua Liu, Panpan Ma, Giovanni Neri, Sirine Elmousli and Amir Khaled Louaar for their valuable contribution to the nanomaterials research axis at UTCBS.

Desmaële, D., Gref, R., and Couvreur, P. (2012). Squalenoylation: a generic platform for nanoparticular drug delivery. J. Controlled Release 161, 609-618. doi: 10.1016/j.jconrel.2011.07.038

Do, H. D., Couillaud, B. M., Doan, B.-T., Corvis, Y., and Mignet, N. (2019). Advances on non-invasive physically triggered nucleic acid delivery from nanocarriers. Adv. Drug Deliv. Rev. 138, 3-17. doi: 10.1016/j.addr.2018.10.006

Fadeel, B. (2019). Hide and seek: nanomaterial interactions with the immune system. Front. Immunol. 10:133. doi: 10.3389/fimmu.2019.00133

Fam, S. Y., Chee, C. F., Yong, C. Y., Ho, K. L., Mariatulqabtiah, A. R., and Tan, W. S. (2020). Stealth coating of nanoparticles in drug-delivery systems. Nanomaterials 10:787. doi: 10.3390/nano10040787

Greenwood, B. (2014). The contribution of vaccination to global health: past, present and future. Philos. Trans. R. Soc. Lond. B. Biol. Sci. 369:20130433. doi: 10.1098/rstb.2013.0433

Imai, S., Hirai, Y., Nagao, C., Sawamoto, M., and Terashima, T. (2018). Programmed self-assembly systems of amphiphilic random copolymers into size-controlled and thermoresponsive micelles in water. Macromolecules 51, 398-409. doi: 10.1021/acs.macromol.7b01918

Journeay, W. S., Suri, S. S., Moralez, J. G., Fenniri, H., and Singh, B. (2008). Rosette nanotubes show low acute pulmonary toxicity in vivo. Int. J. Nanomed. 3, 373-383. doi: 10.2147/ijn.s3489

Journeay, W. S., Suri, S. S., Moralez, J. G., Fenniri, H., and Singh, B. (2009). Macrophage inflammatory response to self-assembling rosette nanotubes. Small 5, 1446-1452. doi: 10.1002/smll.200801717

Klibanov, A. L., Maruyama, K., Torchilin, V. P., and Huang, L. (1990). Amphipathic polyethyleneglycols effectively prolong the circulation time of liposomes. FEBS Lett. 268, 235-237. doi: 10.1016/0014-5793(90)81016-h

Lecuyer, T., Durand, M.-A., Volatron, J., Desmau, M., Lai-Kuen, R., Corvis, Y., et al. (2020). Degradation of znga2o4:Cr3+ luminescent nanoparticles in lysosomal-like medium. Nanoscale 12, 1967-1974. doi: 10.1039/c9nr06867h

Lehn, J.-M. (2015). Perspectives in chemistry - aspects of adaptive chemistry and materials. Angew. Chem. Int. Ed. Engl. 54, 3276-3289. doi: 10.1002/anie.201409399 
Lehn, J.-M. (2017). Supramolecular chemistry: Where from? Where to? Chem. Soc. Rev. 46, 2378-2379. doi: 10.1039/c7cs00115k

Maldiney, T., Bessiere, A., Seguin, J., Teston, E., Sharma, S. K., Viana, B., et al. (2014). The in vivo activation of persistent nanophosphors for optical imaging of vascularization, tumours and grafted cells. Nat. mater. 13, 418-426. doi: $10.1038 /$ nmat3908

Manta, S., Bessodes, M., Bureau Michel, F., Scherman, D., Delalande, A., Pichon, C., et al. (2016). Characterization of positively charged lipid shell microbubbles with tunable resistive pulse sensing (trps) method: a technical note. Ultrasound Med. Biol. 42, 624-630. doi: 10.1016/j.ultrasmedbio.2015.10.010

Manta, S., Renault, G., Delalande, A., Couture, O., Lagoutte, I., Seguin, J., et al. (2017). Cationic microbubbles and antibiotic-free miniplasmid for sustained ultrasound-mediated transgene expression in liver. J. Controlled Release 262, 170-181. doi: 10.1016/j.jconrel.2017.07.015

Martin, B., Seguin, J., Annereau, M., Fleury, T., Laï-Kuen, R., Neri, G., et al. (2020). Preparation of parenteral nanocrystal suspensions of etoposide from the excipient free dry state of the drug to enhance in vivo antitumoral properties. Sci. Rep. 10:18059. doi: 10.1038/s41598-020-74809-z

Meng, X., Stout, D. A., Sun, L., Beingessner, R. L., Fenniri, H., and Webster, T. J. (2013). Novel injectable biomimetic hydrogels with carbon nanofibers and self assembled rosette nanotubes for myocardial applications. J. Biomed. Mater. Res. Part A 101A, 1095-1102. doi: 10.1002/jbm.a.34400

Mignet, N., Seguin, J., Ramos Romano, M., Brulle, L., Touil, Y. S., Scherman, D., et al. (2012). Development of a liposomal formulation of the natural flavonoid fisetin. Int. J. Pharm. 423, 69-76. doi: 10.1016/j.ijpharm.2011.04.066

Mohamed, M. A., Fallahi, A., El-Sokkary, A. M. A., Salehi, S., Akl, M. A., Jafari, A., et al. (2019). Stimuli-responsive hydrogels for manipulation of cell microenvironment: from chemistry to biofabrication technology. Prog. Polym. Sci. 98:101147. doi: 10.1016/j.progpolymsci.2019.101147

Negahdaripour, M., Golkar, N., Hajighahramani, N., Kianpour, S., Nezafat, N., and Ghasemi, Y. (2017). Harnessing self-assembled peptide nanoparticles in epitope vaccine design. Biotechnol. Adv. 35, 575-596. doi: 10.1016/j.biotechadv.2017.05.002

Park, J.-S., Ahn, J.-Y., Lee, S.-H., Lee, H., Han, K.-Y., Seo, H.-S., et al. (2007). Enhanced stability of heterologous proteins by supramolecular self-assembly. Appl. Microbiol. Biotechnol. 75, 347-355. doi: 10.1007/s00253-006-0826-3

Pedersen, S. L., Huynh, T. H., Poschko, P., Fruergaard, A. S., Jarlstad Olesen, M. T., Chen, Y., et al. (2020). Remotely triggered liquefaction of hydrogel materials. ACS Nano 14, 9145-9155. doi: 10.1021/acsnano.0c04522

Prevot, G., Soria, F. N., Thiolat, M.-L., Daniel, J., Verlhac, J. B., Blanchard-Desce, M., et al. (2018). Harnessing lysosomal ph through plga nanoemulsion as a treatment of lysosomal-related neurodegenerative diseases. Bioconjug. Chem. 29, 4083-4089. doi: 10.1021/acs.bioconjchem.8b00697
Puzan, M. L., Legesse, B., Koppes, R. A., Fenniri, H., and Koppes, A. N. (2018). Bioactive organic rosette nanotubes support sensory neurite outgrowth. ACS Biomater. Sci. Eng. 4, 1630-1640. doi: 10.1021/acsbiomaterials.8b00326

Song, S., Chen, Y., Yan, Z., Fenniri, H., and Webster, T. J. (2011). Self-assembled rosette nanotubes for incorporating hydrophobic drugs in physiological environments. Int. J. Nanomed. 6, 101-107. doi: 10.2147/ijn.s11957

Sun, L., Li, D., Hemraz, U. D., Fenniri, H., and Webster, T. J. (2014). Selfassembled rosette nanotubes and poly(2-hydroxyethyl methacrylate) hydrogels promote skin cell functions. J. Biomed. Mater. Res. Part A 102A, 3446-3451. doi: 10.1002/jbm.a.35008

Sun, L., Zhang, L., Hemraz, U. D., Fenniri, H., and Webster, T. J. (2012). Bioactive rosette nanotube-hydroxyapatite nanocomposites improve osteoblast functions. Tissue Eng. Part A 18, 1741-1750. doi: 10.1089/ten.tea.2011.0456

Tan, J. S., Butterfield, D. E., Voycheck, C. L., Caldwell, K. D., and Li, J. T. (1993). Surface modification of nanoparticles by peo/ppo block copolymers to minimize interactions with blood components and prolong blood circulation in rats. Biomaterials 14, 823-833. doi: 10.1016/0142-9612(93)90004-1

Teston, E., Maldiney, T., Marangon, I., Volatron, J., Lalatonne, Y., Motte, L., et al. (2018). Nanohybrids with magnetic and persistent luminescence properties for cell labeling, tracking, in vivo real-time imaging, and magnetic vectorization. Small 14:1800020. doi: 10.1002/smll.201800020

Wren, S., Minelli, C., Pei, Y., and Akhtar, N. (2020). Evaluation of particle size techniques to support the development of manufacturing scale nanoparticles for application in pharmaceuticals. J. Pharm. Sci. 109, 2284-2293. doi: 10.1016/j.xphs.2020.04.001

Zhou, X., Tenaglio, S., Esworthy, T., Hann, S. Y., Cui, H., Webster, T. J., et al. (2020). Three-dimensional printing biologically inspired DNA-based gradient scaffolds for cartilage tissue regeneration. ACS Appl. Mater. Interfaces 12, 33219-33228. doi: 10.1021/acsami.0c07918

Conflict of Interest: VL was employed by the GSK group of companies.

The remaining authors declare that the research was conducted in the absence of any commercial or financial relationships that could be construed as a potential conflict of interest.

Copyright (C) 2020 Lepeltier, Levet, Lee, Mignet, Shen, Fenniri and Corvis. This is an open-access article distributed under the terms of the Creative Commons Attribution License (CC BY). The use, distribution or reproduction in other forums is permitted, provided the original author(s) and the copyright owner(s) are credited and that the original publication in this journal is cited, in accordance with accepted academic practice. No use, distribution or reproduction is permitted which does not comply with these terms. 